\title{
Antiplane Stoneley waves propagating at the interface between two couple stress elastic materials
}

\author{
Andrea Nobili ${ }^{1,2,3}$. Valentina Volpini ${ }^{1,2}$. \\ Cesare Signorini ${ }^{3}$
}

the date of receipt and acceptance should be inserted later

\begin{abstract}
We investigate antiplane Stoneley waves, localized at the discontinuity surface between two half-spaces in perfect contact. Both half-spaces are elastic linear isotropic and possess a microstructure that is described within the theory of couple stress materials with micro-inertia. In contrast to classical elasticity, where antiplane Stoneley waves are never supported and in-plane Stoneley waves exist only inasmuch as the ratio of the shear velocity of the half-spaces is small enough, we find that propagation is possible under broad conditions. However, Stoneley waves only propagate beyond a cuton frequency, for which an explicit expression is provided. For a given frequency above cuton, this expression lends the admissible range of material parameters that allows propagation. In particular, significant contrast between the adjoining materials is possible, provided that Stoneley waves propagate at high-enough frequency. Therefore, micro-inertia plays an important role in determining the features of propagation. Considerations concerning existence and uniqueness of antiplane Stoneley waves are given: it is found that evanescent and decaying/exploding modes are also admitted. Results may be especially useful when accounting for microstructure in Non-Destructive Testing (NDT) and near-surface prospection.
\end{abstract}

Keywords Antiplane Stoneley waves · microstructure · couple-stress · existence and uniqueness

Andrea Nobili

Department of Engineering Enzo Ferrari, University of Modena and Reggio Emilia, via Vivarelli 10, 41125 Modena, Italy

E-mail: andrea.nobili@unimore.it

Valentina Volpini

Research Centre CRICT, via Vivarelli 10, 41125 Modena, Italy

Cesare Signorini

Centre En\&Tech, Tecnopolo, p.le Europa 1, 42124 Reggio Emilia, Italy 


\section{Introduction}

The quest for proving existence of new types of localized waves, similar in nature to Rayleigh waves occurring at a free surface, begun shortly after the discovery of these in 1885 [30]. In 1911, Love [16, p.165] investigated the possibility of waves propagating at the free surface of a layer in perfect contact with a half-space, in an attempt to explain the problematic (from the theoretical standpoint) appearance of shear horizontal Rayleigh waves in seismograms [19]. Later, Stoneley investigated the existence of waves localised at the surface of discontinuity between two materials [28, 29]. As he points out "Whereas, however, Prof. Love's problem is concerned with a disturbance confined chiefly to the free surface, the present paper deals with a wave motion that is greatest at the surface of separation of the two media" [29]. Indeed, these waves go under the name of generalized Rayleigh waves or, quite fittingly, Stoneley waves. Stoneley concludes that "we can definitely assert that when the wave-velocities are not too widely different for the two media, a wave of the Rayleigh type can exist at the interface". This finding is somewhat surprising, in that it allows localized waves to exists only at "weak" boundaries, whereas the exact opposite could be expected. At the time of Stoneley, the main motivation behind the investigation was to determine whether seismic energy could escape at the Gutenberg-Wiechert boundary between the Earth's mantle and the core. Indeed, existence conditions were supposed to correspond to Wiechert conditions, expressing equality of mass density and Lamé moduli across the surface of discontinuity. Precise quantification of the range of existence of Stoneley waves came much later, by Scholte [26]. Scholte conditions are very restrictive for, as pointed out in $[23,11]$, they are satisfied merely by 31 combinations among 900 isotropic materials.

Existence and uniqueness for Stoneley waves have been investigated in the monograph [3, Chap.4], by applying the argument principle to the corresponding Rayleigh function. Extension to anisotropic materials, which admit generalized Stoneley waves, was given by Lin and Musgrave [15] and by Chadwick and Borejko [4] (notably, Chadwick has been Robert Stoneley's research student).

Recently, the role of material microstructure has attracted considerable attention in connection with wave propagation and related phenomena $[22,8,10,27$, $9,20,19]$. One way of encompassing material micro-structure in the models is by means of polar theories, among which couple stress (CS) theory is perhaps the simplest $[17,24]$. Couple stress theory builds on top of classical elasticity (CE) by adding an extra kinematical field, named the micro-rotation. However, in contrast to micro-polar theory, micro-rotation is taken to be entrained by the displacement field [13]. As a result, a length scale is introduced in the system and the theory is no longer self-similar. Consequently, some unphysical results appearing in CE are repaired, such as the non-dispersive nature of bulk and Rayleigh waves and the lack of support for anti-plane (or shear horizontally polarized) Rayleigh and interfacial (Stoneley) waves. It is precisely the last issue that is addressed in this paper.

In recent times, a number of contributions have appeared in the literature concerning propagation of Stoneley waves. In [14], propagation of Stoneley waves is considered within the context of the modified couple stress theory, which is a special case wherein symmetry of the couple stress tensor is enforced [33]. The speed of Stoneley waves guided by a perfect interface between two elastic half-spaces is 
determined analytically in [32] for the case of equal bulk moduli. Consideration of Stoneley waves propagating at a loose interface between two elastic half-spaces is given in [31]. Similarly, guided propagation occurring between two half-spaces in elastically constrained contact is considered in [1]. Since classical elasticity does not support antiplane Stoneley waves, every listed contribution deals with waves polarized in the sagittal plane, to which the term Stoneley waves is traditionally attached. As a notable exception, in [6] antiplane Stoneley waves are shown to occur at the interface between two half-spaces in the presence of surface elasticity.

As illustrated in [19], the presence of the microstructure provides new pathways for energy transport, which take the form of novel wave propagation patterns being supported. In this paper, we show that, in contrast to CE, anti-plane Stoneley waves are supported in CS materials under very general conditions concerning the elastic contrast between the media in contact. However, propagation is only permitted beyond a cuton frequency that is an increasing function of this contrast. Indeed, the role of rotational inertia is especially important for determining the range of admissible parameters for propagation to occur. Consequently, despite its appealing simplicity, the approximation of zero rotation inertia should be avoided. We also discuss existence and uniqueness of Stoneley waves.

Traditionally, Stoneley waves have been exploited in borehole seismics to determine the shear-wave velocities at different depths. Also, thin-film applications are possible, as it is demonstrated in [25]. A pioneering application to Non-Destructive Testing (NDT) is experimentally investigated in [11]. The recent monograph [5] collects 14 real-life case-study applications of surface and near-surface waves, ranging from geophysics to civil engineering, from geotechnics to moonquakes. Today, investigation of Stoneley waves is sustained by modern promising developments in the field of acoustic NDT, as very recently discussed in [12]. This paper aims to add a further tool to the investigator, in the form of anti-plane interfacial waves reflecting the microstructure underneath the continuous media.

\section{Two couple stress elastic half-spaces in perfect contact}

We consider two half-spaces, named $\mathrm{A}$ and $\mathrm{B}$, in perfect contact along a plane surface. We introduce a right-handed Cartesian coordinate system $\left(O, x_{1}, x_{2}, x_{3}\right)$, whose axes are directed along the relevant unit vectors $\left(\boldsymbol{e}_{\mathbf{1}}, \boldsymbol{e}_{\mathbf{2}}, \boldsymbol{e}_{\mathbf{3}}\right)$. The co-ordinate system is located in such a way that the plane $x_{2}=0$ corresponds to the contact surface between A and B, see Fig.1. Both half-spaces possess a microstructure, which is described within the theory of linear couple stress (CS) elasticity. Their relevant properties are henceforth denoted by the superscript $\mathrm{k} \in\{\mathrm{A}, \mathrm{B}\}$.

The stress state in each half-space depends not only on the classical Cauchy force stress tensor $\boldsymbol{s}^{\mathrm{k}}$, but also on the couple stress tensor $\boldsymbol{\mu}^{\mathrm{k}}$. The latter characterises the polar behaviour of the material such that, for any directed surface of unit normal $\boldsymbol{n}^{\mathrm{k}}$, it determines the internal reduced couple vector $\boldsymbol{q}^{\mathrm{k}}$, acting across that surface

$$
\boldsymbol{q}^{\mathrm{k}}=\left(\boldsymbol{\mu}^{\mathrm{k}}\right)^{\mathrm{T}} \boldsymbol{n}^{\mathrm{k}}
$$

where the superscript $\mathrm{T}$ denotes the transposed tensor. $\boldsymbol{s}^{\mathrm{k}}$ is conveniently decomposed into its symmetric and skew-symmetric part

$$
\boldsymbol{s}^{\mathrm{k}}=\boldsymbol{\sigma}^{\mathrm{k}}+\boldsymbol{\tau}^{\mathrm{k}}, \quad \boldsymbol{\sigma}^{\mathrm{k}}=\operatorname{Sym} \boldsymbol{s}^{\mathrm{k}}, \quad \boldsymbol{\tau}^{\mathrm{k}}=\operatorname{Skw} \boldsymbol{s}^{\mathrm{k}},
$$




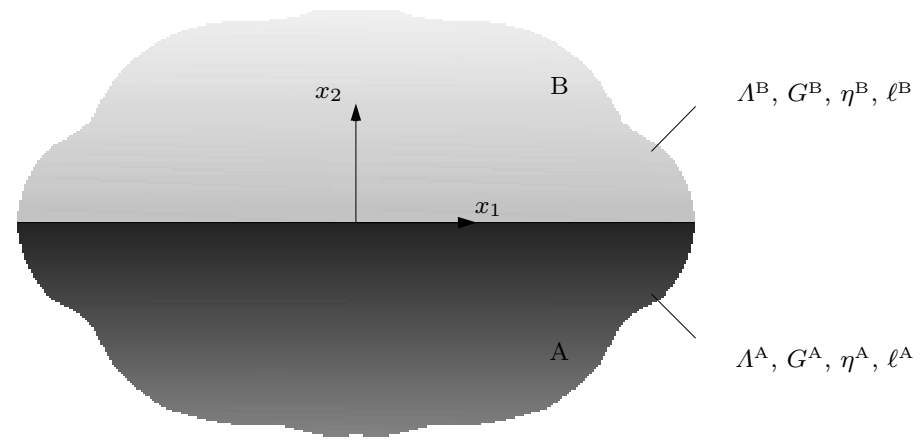

Fig. 1 Schematics, mechanical and microstructural properties of the two half-spaces A and B

whereas $\boldsymbol{\mu}^{\mathrm{k}}$ is decomposed in the deviatoric and spherical part

$$
\mu^{\mathrm{k}}=\boldsymbol{\mu}_{\mathrm{D}}^{\mathrm{k}}+\boldsymbol{\mu}_{\mathrm{S}}^{\mathrm{k}}
$$

Specifically, the latter reads

$$
\boldsymbol{\mu}_{\mathrm{S}}^{\mathrm{k}}=\frac{1}{3}\left(\boldsymbol{\mu}^{\mathrm{k}} \cdot \mathbf{1}\right) \mathbf{1}
$$

wherein a dot denotes the scalar product and $\mathbf{1}$ is the rank-2 identity tensor.

In each half-space, occupying the volume $\mathcal{B}^{\mathrm{k}}$, the internal virtual work may be expressed as follows (see, e.g., [13, 22]):

$$
\int_{\mathcal{B}^{\mathrm{k}}}\left(\boldsymbol{\sigma}^{\mathrm{k}} \cdot \operatorname{grad} \boldsymbol{u}^{\mathrm{k}}+\boldsymbol{\mu}^{\mathrm{k}} \cdot \operatorname{grad}^{\mathrm{T}} \boldsymbol{\varphi}^{\mathrm{k}}\right) \mathrm{d} V
$$

where grad denotes the gradient operator. Here, $\boldsymbol{u}^{\mathrm{k}}$ and $\boldsymbol{\varphi}^{\mathrm{k}}$ are the displacement and the micro-rotation vector fields. Within the theory of CS materials (see, for instance, [13]), the displacement field $\boldsymbol{u}^{\mathrm{k}}$ and the micro-rotation field $\boldsymbol{\varphi}^{\mathrm{k}}$ are related by

$$
\varphi^{\mathrm{k}}=\frac{1}{2} \operatorname{curl} \boldsymbol{u}^{\mathrm{k}}, \quad \Leftrightarrow \quad \varphi_{i}^{\mathrm{k}}=\frac{1}{2} \mathbb{E}_{i j s}^{\mathrm{k}} u_{s, j}^{\mathrm{k}}
$$

where it is understood that a subscript comma denotes partial differentiation, i.e. $u_{i, j}=(\operatorname{grad} \boldsymbol{u})_{i j}=\partial u_{i} / \partial x_{j}$, and $\mathbb{E}$ is the rank-3 Levi-Civita (permutation) tensor.

By standard arguments of linear elasticity, the displacement field is related to the linear strain tensor $\varepsilon^{k}$ through

$$
\varepsilon^{\mathrm{k}}=\text { Sym grad } \boldsymbol{u}^{\mathrm{k}}
$$


and this is work-conjugated to $\boldsymbol{\sigma}$ (see (5)). In similar manner, we introduce the torsion-flexure (or wryness) tensor

$$
\chi^{\mathrm{k}}=\operatorname{grad} \varphi^{\mathrm{k}}
$$

which is work-conjugated to $\boldsymbol{\mu}^{\mathrm{k}}$.

By combining Eqs. $(5,6)$ and (8), we infer

$$
\chi^{\mathrm{k}}=\chi_{\mathrm{D}}^{\mathrm{k}} \stackrel{\text { Eq.(5) }}{\longrightarrow} \mu^{\mathrm{k}}=\boldsymbol{\mu}_{\mathrm{D}}^{\mathrm{k}}
$$

that is, the torsion-flexure tensor is purely deviatoric and the couple stress tensor, to all intents and purposes, may be replaced by the sole deviatoric part $\boldsymbol{\mu}_{\mathrm{D}}$. To light notation, hereinafter we write $\boldsymbol{\mu}^{\mathrm{k}}$ with the understanding that $\boldsymbol{\mu}_{\mathrm{D}}^{\mathrm{k}}$ is meant.

\subsection{Constitutive prescriptions}

We assume hyperelastic isotropic material behaviour for both the half-spaces A and B. Accordingly, four material parameters are introduced for each space, namely the classical Lamé moduli, $\Lambda^{\mathrm{k}}>0$ and $G^{\mathrm{k}}>0$, alongside $\ell^{\mathrm{k}}>0$ and $-1<\eta^{\mathrm{k}}<1$, characterising the microstructure. More specifically, $\eta^{\mathrm{k}}$ plays a role similar to that of the Poisson's ratio, whereas $\ell^{k}$ is defined as the characteristic length of the microstructure. Both parameters may be related to the characteristic length in bending and in torsion, $\ell_{b}^{\mathrm{k}}$ and $\ell_{t}^{\mathrm{k}}$, respectively, through the following expressions $[24,20]$

$$
\ell_{b}^{\mathrm{k}}=\ell^{\mathrm{k}} / \sqrt{2}
$$

$$
\ell_{t}^{\mathrm{k}}=\ell^{\mathrm{k}} \sqrt{1+\eta^{\mathrm{k}}} .
$$

In polycrystalline metals, $\eta=-1$ and the length in torsion $\ell_{t}$ vanishes, while the case

$$
\eta=0 \quad \Rightarrow \quad \ell_{t}=\ell=\sqrt{2} \ell_{b}
$$

relates to the so-called strain gradient effect, taken into account in [34].

Following [13], in each half-space we define a free-energy density $U^{\mathrm{k}}\left(\varepsilon^{\mathrm{k}}, \chi^{\mathrm{k}}\right)$, such that the following constitutive relations hold:

$$
\begin{array}{lll}
\boldsymbol{\sigma}^{\mathrm{k}}=\frac{\partial U^{\mathrm{k}}}{\partial \boldsymbol{\varepsilon}^{\mathrm{k}}} & \Rightarrow & \boldsymbol{\sigma}^{\mathrm{k}}=2 G^{\mathrm{k}} \varepsilon^{\mathrm{k}}+\Lambda^{\mathrm{k}}\left(\operatorname{tr} \boldsymbol{\varepsilon}^{\mathrm{k}}\right) \mathbf{1}, \\
\boldsymbol{\mu}^{\mathrm{k}}=\frac{\partial U^{\mathrm{k}}}{\partial \boldsymbol{\chi}^{\mathrm{k}}} & \Rightarrow & \boldsymbol{\mu}^{\mathrm{k}}=2 G^{\mathrm{k}}\left(\ell^{\mathrm{k}}\right)^{2}\left[\left(\boldsymbol{\chi}^{\mathrm{k}}\right)^{\mathrm{T}}+\eta^{\mathrm{k}} \boldsymbol{\chi}^{\mathrm{k}}\right] .
\end{array}
$$

We observe that, in the special case $\eta=1$, the CS theory at hand specializes to the modified CS theory of elasticity introduced in [33], in which $\ell_{b}=\ell_{t} / 2=\ell / \sqrt{2}$ and the CS tensor is symmetric, owing to a postulated balance law concerning equilibrium of the torque of torques. 
In each half-space, the equations of motion, in the absence of body forces, read

$$
\begin{aligned}
\operatorname{div} \boldsymbol{s}^{\mathrm{k}} & =\rho^{\mathrm{k}} \ddot{\boldsymbol{u}}^{\mathrm{k}}, \\
2 \operatorname{axial} \boldsymbol{\tau}^{\mathrm{k}}+\operatorname{div} \boldsymbol{\mu}^{\mathrm{k}} & =J^{\mathrm{k}} \ddot{\boldsymbol{\varphi}}^{\mathrm{k}},
\end{aligned}
$$

having indicated time differentiation with a superposed dot. Here, $\rho^{\mathrm{k}}$ and $J^{\mathrm{k}} \geq 0$ are the mass density and the rotational inertia per unit volume, respectively. Besides, $(\text { axial } \tau)_{i}^{\mathrm{k}}=\frac{1}{2} \mathbb{E}_{i j k}^{\mathrm{k}} \tau_{k j}^{\mathrm{k}}$ denotes the axial vector attached to the skew-symmetric tensor $\boldsymbol{\tau}$. Naturally, Eqs.(12) reduce to the equations of classical elasticity $(\mathrm{CE})$, upon setting

$$
\ell=0 \quad \text { and } \quad J=0 \Rightarrow \boldsymbol{\mu}=\boldsymbol{\tau}=\mathbf{0} .
$$

\subsection{Antiplane shear deformations}

We assume antiplane shear deformations, such that, in each half-space, the displacement field $\boldsymbol{u}^{\mathrm{k}}$ reduces to the out-of-plane component only

$$
u_{3}^{\mathrm{k}}\left(x_{1}, x_{2}, t\right)
$$

and there is no dependence on the $x_{3}$ co-ordinate. Within this framework, according to Eqs. $(6,7,8)$, the only non-vanishing components of the strain and of the torsion-flexure tensors are

$$
\begin{aligned}
\varepsilon_{13}^{\mathrm{k}} & =\frac{1}{2} u_{3,1}^{\mathrm{k}}, & \varepsilon_{23}^{\mathrm{k}} & =\frac{1}{2} u_{3,2}^{\mathrm{k}}, \\
\varphi_{1}^{\mathrm{k}} & =\frac{1}{2} u_{3,2}^{\mathrm{k}}, & \varphi_{2}^{\mathrm{k}} & =-\frac{1}{2} u_{3,1}^{\mathrm{k}}, \\
\chi_{11}^{\mathrm{k}} & =-\chi_{22}^{\mathrm{k}}=\frac{1}{2} u_{3,12}^{\mathrm{k}}, & \chi_{21}^{\mathrm{k}} & =-\frac{1}{2} u_{3,11}^{\mathrm{k}}, \quad \chi_{12}^{\mathrm{k}}=\frac{1}{2} u_{3,22}^{\mathrm{k}} .
\end{aligned}
$$

Besides, accounting for the constitutive relations (11) and in light of (13), we get

$$
\begin{array}{ll}
\sigma_{13}^{\mathrm{k}}=G^{\mathrm{k}} u_{3,1}^{\mathrm{k}}, & \sigma_{23}^{\mathrm{k}}=G^{\mathrm{k}} u_{3,2}^{\mathrm{k}}, \\
\mu_{11}^{\mathrm{k}}=-\mu_{22}^{\mathrm{k}}=G^{\mathrm{k}}\left(\ell^{\mathrm{k}}\right)^{2}\left(1+\eta^{\mathrm{k}}\right) u_{3,12}^{\mathrm{k}}, & \mu_{21}^{\mathrm{k}}=G^{\mathrm{k}}\left(\ell^{\mathrm{k}}\right)^{2}\left(u_{3,22}^{\mathrm{k}}-\eta u_{3,11}^{\mathrm{k}}\right), \\
\mu_{12}^{\mathrm{k}}=-G^{\mathrm{k}}\left(\ell^{\mathrm{k}}\right)^{2}\left(u_{3,11}^{\mathrm{k}}-\eta^{\mathrm{k}} u_{3,22}^{\mathrm{k}}\right), &
\end{array}
$$

in which the Lamé constant $\Lambda^{\mathrm{k}}$ does not play any role. The balance equations $(12)$ reduce to

$$
\begin{aligned}
\sigma_{13,1}^{\mathrm{k}}+\sigma_{23,2}^{\mathrm{k}}+\tau_{13,1}^{\mathrm{k}}+\tau_{23,2}^{\mathrm{k}} & =\rho^{\mathrm{k}} \ddot{u}_{3}^{\mathrm{k}}, \\
\mu_{11,1}^{\mathrm{k}}+\mu_{21,2}^{\mathrm{k}}+2 \tau_{23}^{\mathrm{k}} & =J^{\mathrm{k}} \ddot{\varphi}_{1}^{\mathrm{k}}, \\
\mu_{12,1}^{\mathrm{k}}+\mu_{22,2}^{\mathrm{k}}-2 \tau_{13}^{\mathrm{k}} & =J^{\mathrm{k}} \ddot{\varphi}_{2}^{\mathrm{k}} .
\end{aligned}
$$

Now, we solve Eq.(12b) for $\tau^{\mathrm{k}}$

$$
\boldsymbol{\tau}^{\mathrm{k}}=\frac{1}{2} \mathbb{E}^{\mathrm{k}}\left(\operatorname{div} \boldsymbol{\mu}^{\mathrm{k}}-J^{\mathrm{k}} \ddot{\varphi}^{\mathrm{k}}\right)
$$


and substitute Eqs. (13b) and (14) into (16), to obtain (see [18])

$$
\begin{aligned}
\tau_{13}^{\mathrm{k}} & =-\frac{1}{2} G^{\mathrm{k}}\left(\ell^{\mathrm{k}}\right)^{2} \hat{\triangle} u_{3,1}^{\mathrm{k}}+\frac{J^{\mathrm{k}}}{4} \ddot{u}_{3,1}^{\mathrm{k}}, \\
\tau_{23}^{\mathrm{k}} & =-\frac{1}{2} G^{\mathrm{k}}\left(\ell^{\mathrm{k}}\right)^{2} \hat{\triangle} u_{3,2}^{\mathrm{k}}+\frac{J^{\mathrm{k}}}{4} \ddot{u}_{3,2}^{\mathrm{k}},
\end{aligned}
$$

for media A, and

$$
p_{3}^{\mathrm{B}}=-\left(s_{23}^{\mathrm{B}}+\frac{1}{2} \mu_{22,1}^{\mathrm{B}}\right), \quad \bar{q}_{1}^{\mathrm{B}}=-\mu_{21}^{\mathrm{B}}, \quad \text { with } \quad \bar{q}_{2}^{\mathrm{B}}=0,
$$

${ }_{167}$ for media B. 


\section{Nondimensional equations and time-harmonic solution}

3.1 Nondimensional form of governing equations

We are now in a position to bring in nondimensional form the governing equations of Sec.2, introducing suitable normalizing quantities. Although the problem is symmetric under $A \leftrightarrow B$ inversion, we prefer, for the sake of simplicity, to normalize against either half-space, say A. Thus,

$$
\boldsymbol{\xi}=\frac{\boldsymbol{x}}{\Theta \ell^{\mathrm{A}}}
$$

is the new dimensionless set of coordinates. The quantity $\Theta \ell^{\mathrm{A}}$ is a reference length that is proportional, through the yet-unspecified factor $\Theta$, to the microstructure characteristic length for the half-space A. Similarly, we introduce the reference time $T^{\mathrm{A}}=\ell^{\mathrm{A}} / c_{\mathrm{s}}^{\mathrm{A}}$ and let the dimensionless time

$$
\tau=\frac{t}{T^{\mathrm{A}}} .
$$

Here, $c_{\mathrm{S}}^{\mathrm{A}}=\sqrt{G^{\mathrm{A}} / \rho^{\mathrm{A}}}$ is the shear wave speed of CE for material A. In a similar fashion, $T^{\mathrm{B}}=\ell^{\mathrm{B}} / c_{\mathrm{S}}^{\mathrm{B}}$ is the reference time and $c_{s}^{\mathrm{B}}=\sqrt{G^{\mathrm{B}} / \rho^{\mathrm{B}}}$ the shear wave speed of $\mathrm{CE}$ for material $\mathrm{B}$. It proves convenient to introduce the ratios

$$
\begin{aligned}
& \beta=\frac{\ell^{\mathrm{B}}}{\ell^{\mathrm{A}}}, \\
& v=\frac{T^{\mathrm{A}}}{T^{\mathrm{B}}},
\end{aligned}
$$

whereupon $v \beta=c_{\mathrm{s}}{ }^{\mathrm{B}} / c_{\mathrm{S}}{ }^{\mathrm{A}}$. We observe that the limiting case in which the halfspace $\mathrm{B}$ is constituted by classical elastic isotropic material, i.e. in the absence of microstructure for B, can still be retrieved by taking

$$
v \rightarrow+\infty, \beta \rightarrow 0, \quad \text { s.t. } \beta v<\infty .
$$

The corresponding classical limit for material A cannot be accessed directly on account of the performed nondimensionalization, although this is no limitation for $\mathrm{A}$ and $\mathrm{B}$ are interchangeable

Substituting the thus introduced nondimensional variables in Eq.(18) lends the nondimensional governing equations

$$
\triangle u_{3}^{\mathrm{A}}-2 \Theta^{2} \triangle u_{3}^{\mathrm{A}}-2 \Theta^{4}\left[\frac{\left(\ell_{0}^{\mathrm{A}}\right)^{2}}{\Theta^{2}} \Delta u_{3, \tau \tau}^{\mathrm{A}}-u_{3, \tau \tau}^{\mathrm{A}}\right]=0,
$$

$$
\triangle \triangle u_{3}^{\mathrm{B}}-2 \frac{\Theta^{2}}{\beta^{2}} \triangle u_{3}^{\mathrm{B}}-2 \Theta^{4}\left[\frac{\left(\ell_{0}^{\mathrm{B}}\right)^{2}}{\Theta^{2} v^{2} \beta^{2}} \Delta u_{3, \tau \tau}^{\mathrm{B}}-\frac{1}{v^{2} \beta^{4}} u_{3, \tau \tau}^{\mathrm{B}}\right]=0,
$$

holding in $\mathrm{A}$ and $\mathrm{B}$, respectively. Here, the symbol $\triangle$ indicates the two-dimensional Laplace operator with respect to $\xi_{1}$ and $\xi_{2}$, whereas the dimensionless parameters $\ell_{0}^{\mathrm{k}}$ are defined as (see also $\left.[18,20]\right)$ :

$$
\ell_{0}^{\mathrm{k}}=\frac{\ell_{d}^{\mathrm{k}}}{\ell^{\mathrm{k}}} \quad \text { with } \quad \ell_{d}^{\mathrm{k}}=\frac{1}{2} \sqrt{\frac{J^{\mathrm{k}}}{\rho^{\mathrm{k}}}}
$$

We note that $\ell_{d}^{\mathrm{k}}$ is proportional to the dynamic characteristic length introduced in [27]. 
3.2 Time-harmonic solution

We consider time-harmonic and straight-crested antiplane waves moving in the sagittal plane $\left(\xi_{1}, \xi_{2}\right)$,

$$
u_{3}^{k}\left(\xi_{1}, \xi_{2}, \tau\right)=W^{\mathrm{k}}\left(\xi_{1}, \xi_{2}\right) \exp (-\imath \Omega \tau)
$$

where $\imath$ is the imaginary unit and $\Omega=\omega T^{\mathrm{A}}>0$ indicates the nondimensional time frequency. Substituting the solution form (25) into Eqs.(24), we obtain the pair of meta-biharmonic partial differential equations (PDEs)

$$
\begin{array}{r}
{\left[\triangle \triangle-2\left(1-\left(\ell_{0}^{\mathrm{A}}\right)^{2} \Omega^{2}\right) \Theta^{2} \triangle-2 \Omega^{2} \Theta^{4}\right] W^{\mathrm{A}}=0,} \\
{\left[\triangle \triangle-2\left(1-\frac{\left(\ell_{0}^{\mathrm{B}}\right)^{2}}{v^{2}} \Omega^{2}\right) \frac{\Theta^{2}}{\beta^{2}} \Delta-2 \Omega^{2} \frac{\Theta^{4}}{v^{2} \beta^{4}}\right] W^{\mathrm{B}}=0 .}
\end{array}
$$

Eq.(26a) may be factored out as [20]

$$
\left(\triangle+\delta^{2}\right)(\triangle-1) W^{\mathrm{A}}=0,
$$

provided that we make the proper choice for $\Theta$. Indeed, applying Vieta's theorem to Eqs.(26a,27), we get

$$
\delta^{2}=2 \Omega^{2} \Theta^{4}, \quad \text { and } \quad 1-\delta^{2}=2 \Theta^{2}\left(1-\left(\ell_{0}^{\mathrm{A}}\right)^{2} \Omega^{2}\right),
$$

whence we need to let the dimensionless factor $\Theta$ as the positive root of the biquadratic equation

$$
2 \Omega^{2} \Theta^{4}+2\left[1-\left(\ell_{0}^{\mathrm{A}}\right)^{2} \Omega^{2}\right] \Theta^{2}-1=0,
$$

that is

$$
\Theta^{2}=\frac{\sqrt{\left(1-\left(\ell_{0}^{\mathrm{A}}\right)^{2} \Omega^{2}\right)^{2}+2 \Omega^{2}}-1+\left(\ell_{0}^{\mathrm{A}}\right)^{2} \Omega^{2}}{2 \Omega^{2}} .
$$

It is easily seen that $\Theta$ is a bounded function of $\Omega$, while $\delta$ is the wavenumber of shear horizontal ( $\mathrm{SH}$ ) travelling bulk waves. The latter may be rewritten as

$$
\delta=2 \delta_{\mathrm{cr}} \Theta^{2}
$$

having let

$$
\ell_{0 \mathrm{cr}}^{\mathrm{A}}=\frac{1}{\sqrt{2}}, \quad \text { and } \quad \delta_{\mathrm{cr}}=\ell_{0 \mathrm{cr}}^{\mathrm{A}} \Omega=\frac{\Omega}{\sqrt{2}} .
$$

Combining Eqs.(28) and (29), we obtain the connection

$$
\delta\left(\Omega, \ell_{0}^{\mathrm{A}}\right)=\frac{1}{2 \delta_{\mathrm{cr}}}\left[\sqrt{\left(1-\left(\ell_{0}^{\mathrm{A}}\right)^{2} \Omega^{2}\right)^{2}+2 \Omega^{2}}-1+\left(\ell_{0}^{\mathrm{A}}\right)^{2} \Omega^{2}\right] .
$$

This connection becomes simply $\ell_{0}^{\mathrm{A}}=\ell_{0}^{\mathrm{cr}} \mathrm{A}$ the special case $\delta=\delta_{\mathrm{cr}}$. In general, Eq.(30) may be inverted to yield $\Omega$ as a function of $\delta$, and, in the special case $\ell_{0}^{\mathrm{A}}=\ell_{0 \mathrm{cr}}^{\mathrm{A}}$, this relationship is linear. However, when rotational inertia is zero, that is for $\ell_{0}^{\mathrm{A}}=0$, a positive frequency $\Omega$ exists only within the interval $\delta \in(-1,1)$. 
With such definitions, Eq.(26b) may be factored as

$$
\left(\triangle+\delta_{1}^{2}\right)\left(\triangle-\delta_{2}^{2}\right) W^{\mathrm{B}}=0
$$

where we have let the dimensionless wavenumbers for bulk travelling and bulk evanescent waves in medium B

$$
\delta_{1}^{2}=\frac{\delta \psi}{\beta^{2} v^{2}}, \quad \delta_{2}^{2}=\frac{\delta}{\beta^{2} \psi} .
$$

In particular, $\psi$ may be interpreted as a generalization to material $\mathrm{B}$ of the parameter $\delta$

$$
\psi=v \delta\left(\frac{\Omega}{v}, \ell_{0}^{\mathrm{B}}\right)=\frac{v^{2}}{2 \delta_{\mathrm{cr}}}\left[\sqrt{\left(1-\left(\ell_{0}^{\mathrm{B}}\right)^{2} \frac{\Omega^{2}}{v^{2}}\right)^{2}+2 \frac{\Omega^{2}}{v^{2}}}-1+\left(\ell_{0}\right)^{\mathrm{B}} \frac{\Omega^{2}}{v^{2}}\right] .
$$

Indeed, in the special case of a single homogeneous full-space, that is for $\beta=v=1$, we have $\psi=\delta$. The following asymptotics hold

$$
\begin{array}{llrl}
\psi & \rightarrow \sqrt{2} \Omega\left(\ell_{0}{ }^{\mathrm{B}}\right)^{2}+O\left(v^{2}\right), & \text { as } & v \rightarrow 0, \\
\psi \rightarrow \delta_{\mathrm{cr}}+O\left(v^{-2}\right), & \text { as } & v \rightarrow+\infty .
\end{array}
$$

We introduce the shorthands

$$
\left(\kappa_{1}, \kappa_{2}\right)=\beta v\left(\delta_{1}, \delta_{2}\right)
$$

whereby we obtain the bulk travelling and bulk evanescent (dimensional) wavespeeds for material B

$$
c_{\mathrm{SH}}^{\mathrm{B}}=\frac{\Omega \Theta}{\kappa_{1}} c_{\mathrm{S}}^{\mathrm{B}}, \quad c_{\mathrm{iSH}}^{\mathrm{B}}=\frac{\Omega \Theta}{\kappa_{2}} c_{\mathrm{S}}^{\mathrm{B}} .
$$

The corresponding wavenumbers $\delta_{1,2}$ may be used in (33) instead of $\kappa_{1,2}$ when expressing the corresponding bulk wavespeeds in terms of $c_{\mathrm{S}}^{\mathrm{A}}$.

In the absence of microstructure for B, that is in the limit (23), we have $\kappa_{1} \rightarrow$ $\sqrt{\delta \delta_{\mathrm{cr}}}, \kappa_{2} / v \rightarrow \sqrt{\delta / \delta_{\mathrm{cr}}}$ and we retrieve the classical bulk $\mathrm{SH}$ wavespeed

$$
c_{\mathrm{SH}}^{\mathrm{B}} \rightarrow \frac{\Omega \Theta}{\sqrt{\delta \delta_{\mathrm{cr}}}} c_{\mathrm{s}}^{\mathrm{B}}=c_{\mathrm{s}}^{\mathrm{B}}
$$

Conversely, evanescent bulk waves become stationary,

$$
c_{\mathrm{iSH}}^{\mathrm{B}}=\frac{\Omega \Theta}{v \sqrt{\delta / \delta_{\mathrm{cr}}}} c_{\mathrm{S}}^{\mathrm{B}} \rightarrow 0,
$$

and (31) factors out in the product of the Laplacian with the Helmholtz operator

$$
\triangle\left(\triangle+\delta \delta_{\mathrm{cr}} \frac{c_{\mathrm{s}}^{\mathrm{A} 2}}{c_{\mathrm{s}}^{\mathrm{B}} 2}\right) W^{\mathrm{B}}=0 .
$$

Consequently, the solution is given by two terms, the first providing the timeharmonic solution of CE and the second being an harmonic function [20]. 
For the boundary conditions, Eqs.(21a) take on the form

$$
\begin{aligned}
& p_{3}^{\mathrm{A}}=-\frac{G^{\mathrm{A}}}{2 \Theta^{3}}\left[\left(\delta^{2}-1\right) W_{, 2}^{\mathrm{A}}+\left(\eta^{\mathrm{A}}+2\right) W_{, 112}^{\mathrm{A}}+W_{, 222}^{\mathrm{A}}\right], \\
& \bar{q}_{1}^{\mathrm{A}}=\frac{G^{\mathrm{A}} l^{\mathrm{A}}}{\Theta^{2}}\left(W_{, 22}^{\mathrm{A}}-\eta W_{, 11}^{\mathrm{A}}\right),
\end{aligned}
$$

while Eqs.(21b) become

$$
\begin{aligned}
& p_{3}^{\mathrm{B}}=-\frac{G^{\mathrm{B}}}{2 \Theta^{3}}\left\{\beta^{2}\left[\left(\eta^{\mathrm{B}}+2\right) W_{, 112}^{\mathrm{B}}+W_{, 222}^{\mathrm{B}}\right]+\frac{\kappa_{2}^{2}}{v^{2}}\left(\frac{\psi^{2}}{v^{2}}-1\right) W_{, 2}^{\mathrm{B}}\right\}, \\
& \bar{q}_{1}^{\mathrm{B}}=\frac{G^{\mathrm{B}} \ell^{\mathrm{B}}}{\Theta^{2}} \beta^{2}\left(W_{, 22}^{\mathrm{B}}-\eta^{\mathrm{B}} W_{, 11}^{\mathrm{B}}\right) .
\end{aligned}
$$

In the absence of microstructure for B, Eqs.(35) reproduce the classical result

$$
p_{3}^{\mathrm{B}}=\frac{G^{\mathrm{B}}}{\Theta} W^{\mathrm{B}}, 2, \quad \text { and } \quad \bar{q}_{1}^{\mathrm{B}}=0,
$$

expressing the absence of the reduced couple-stress vector and the force traction vector being simply the product of the shear modulus with the shear deformation.

\section{Antiplane Stoneley waves}

For guided waves propagating along the interface $\xi_{2}=0$, we take

$$
W^{\mathrm{k}}\left(\xi_{1}, \xi_{2}\right)=\ell^{\mathrm{A}} w^{\mathrm{k}}\left(\xi_{2}\right) \exp \left(\imath \kappa \xi_{1}\right)
$$

wherein we have introduced the shorthand $\kappa=\Theta K$, with $K=k \ell^{\mathrm{A}}$ denoting the dimensionless (spatial) wavenumber in the propagation direction $\xi_{1}$. Moreover, we define the dimensional phase speed in the propagation direction

$$
c=\frac{\omega}{k}=\frac{\Omega}{\kappa} \Theta c_{\mathrm{s}}^{\mathrm{A}} .
$$

The general decaying solution of Eq.(27) is

$$
w^{\mathrm{A}}\left(\xi_{2}\right)=a_{1} \exp \left(A_{1} \xi_{2}\right)+a_{2} \exp \left(A_{2} \xi_{2}\right)
$$

and for Eq.(31)

$$
w^{\mathrm{B}}\left(\xi_{2}\right)=b_{1} \exp \left(-B_{1} \xi_{2}\right)+b_{2} \exp \left(-B_{2} \xi_{2}\right),
$$

where the coefficients $a_{1,2}$ and $b_{1,2}$ are four amplitudes to be determined. Here, we have let the decay indices

$$
A_{1}=\sqrt{\kappa^{2}-\delta^{2}}, \quad A_{2}=\sqrt{\kappa^{2}+1},
$$

for material A, and, similarly,

$$
B_{1}=\sqrt{\kappa^{2}-\delta_{1}^{2}}, \quad B_{2}=\sqrt{\kappa^{2}+\delta_{2}^{2}},
$$

for material B. Here, the square root is made definite by taking the branch that corresponds to the positive square root of any positive real argument (equivalently, 
we may demand that $A_{1,2}$ and $B_{1,2} \rightarrow \sqrt{p}$, as $\kappa=p \rightarrow+\infty$ real and positive). Branch cuts for the square root are taken parallel to the imaginary axis in antisymmetric fashion, see the discussion in $[21, \S 1.1]$. By this choice, the square roots $A_{2}$ and $B_{2}$ are positive real along the whole real axis, while $A_{1}$ (and $B_{1}$ ) is positive real outside the interval $|\kappa|<\delta$ (respectively $|\kappa|<\delta_{1}$ ) and purely imaginary inside (see [20]). Also, $A_{1}$ and $B_{1}$ are purely imaginary on the imaginary axis, while $A_{2}$ (and $B_{2}$ ) is positive real inside $|\kappa|<1$ (respectively $|\kappa|<\delta_{2}$ ) and purely imaginary outside, with opposite sign on either side of the cuts. Consequently, continuation by Schwarz's reflection principle $f\left(s^{*}\right)=f^{*}(s)$ is warranted outside the interval $|\kappa|<\delta$ (respectively $|\kappa|<\delta_{1}$ ) and by its opposite, $f\left(s^{*}\right)=-f^{*}(s)$, inside. Here, a superscript asterisk denotes complex conjugation, i.e. given $s=\Re(s)+\imath \Im(s)$, we have $s^{*}=\Re(s)-\imath \Im(s)$. It is emphasized that this is not the choice for the cuts taken in [3], where a finite cut is considered instead. However, according to this choice, we get an odd real-valued function along the real axis, and this jeopardizes decay. At any rate, Rayleigh and Stoneley waves need to be slower than the slowest bulk mode. Besides, it is easily proved that

$$
A_{1}<A_{2}, \quad B_{1}<B_{2}, \quad \text { for } \kappa \in \mathbb{R} .
$$

Clearly, $\imath A_{1,2}$ and $\imath B_{1,2}$ are the (dimensionless) wavenumbers in the thickness direction $\xi_{2}$, in the relevant material. Consideration of the dimensional wavenumbers squared

$$
\begin{aligned}
& -\frac{A_{1}^{2}}{\left(\ell^{\mathrm{A}}\right)^{2} \Theta^{2}}=-k^{2}+\frac{\delta^{2}}{\left(\ell^{\mathrm{A}}\right)^{2} \Theta^{2}}=-k^{2}+\frac{1}{\left(\ell^{\mathrm{A}}\right)^{2}}\left(\sqrt{1+2\left(\ell^{\mathrm{A}}\right)^{2} \omega^{2}\left(c_{\mathrm{S}}^{\mathrm{A}}\right)^{-2}}-1\right), \\
& -\frac{A_{2}^{2}}{\left(\ell^{\mathrm{A}}\right)^{2} \Theta^{2}}=-k^{2}-\frac{1}{\left(\ell^{\mathrm{A}}\right)^{2} \Theta^{2}}=-k^{2}-\frac{1}{\left(\ell^{\mathrm{A}}\right)^{2}}\left(\sqrt{1+2\left(\ell^{\mathrm{A}}\right)^{2} \omega^{2}\left(c_{\mathrm{S}}^{\mathrm{A}}\right)^{-2}}+1\right),
\end{aligned}
$$

matches favourably with the corresponding results given in [7]. As discussed in [19], for $\kappa>\delta$, the solution (38) corresponds to a localized travelling wave moving slower than the corresponding bulk wave in material A. Similarly, for $\kappa>\delta_{1}$, the solution (39) corresponds to a localized travelling wave moving slower than the corresponding bulk wave in material $\mathrm{B}$.

\subsection{Rayleigh function: existence of the Rayleigh zeros}

We define the general form of the Rayleigh function

$$
R_{0}\left(\kappa, \lambda_{1}, \lambda_{2}, \eta\right)=\left(\eta \kappa^{2}-\lambda_{1} \lambda_{2}\right)^{2}-\lambda_{1} \lambda_{2}\left(\lambda_{1}+\lambda_{2}\right)^{2},
$$

that is valid for either half-space, provided that we substitute $\lambda_{1,2}$ with the relevant decay index along $\xi_{2}$. Indeed, for the half-space $\mathrm{A}$, we have $\lambda_{1,2}=A_{1,2}, \eta=\eta^{A}$, and we get $R_{0}^{\mathrm{A}}(\kappa)=R_{0}\left(\kappa, A_{1}, A_{2}, \eta^{\mathrm{A}}\right)$. Multiplication by $\left(A_{1}-A_{2}\right)$ lends the Rayleigh function in the form already exposed in $[19,20]$

$$
R^{\mathrm{A}}(\kappa)=\left(A_{1}-A_{2}\right) R_{0}^{\mathrm{A}}(\kappa)=\left[\left(1+\eta^{\mathrm{A}}\right) \kappa^{2}+1\right]^{2} A_{1}-\left[\left(1+\eta^{\mathrm{A}}\right) \kappa^{2}-\delta^{2}\right]^{2} A_{2} .
$$

In similar fashion, for the half-space $\mathrm{B}$, we have $R_{0}^{\mathrm{B}}(\kappa)=R_{0}\left(\kappa, B_{1}, B_{2}, \eta^{\mathrm{B}}\right)$ and multiplication by $\left(B_{1}-B_{2}\right)$ gives

$$
R^{\mathrm{B}}(\kappa)=\left[\left(\eta^{\mathrm{B}}+1\right) \kappa^{2}+\delta_{2}^{2}\right]^{2} B_{1}-\left[\left(\eta^{\mathrm{B}}+1\right) \kappa^{2}-\delta_{1}^{2}\right]^{2} B_{2},
$$




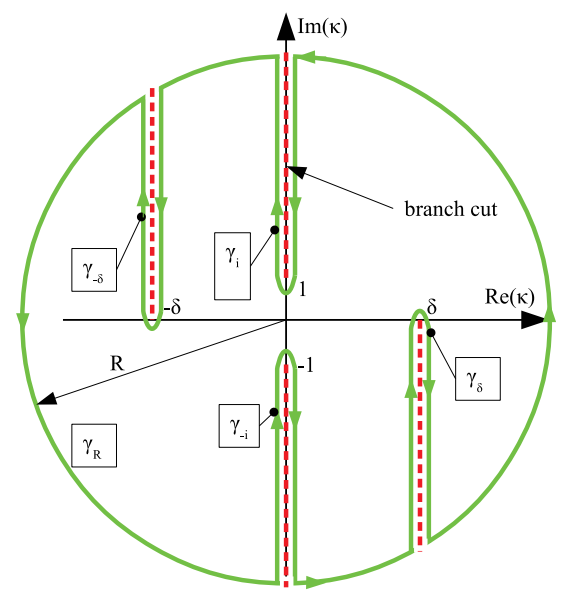

Fig. 2 Simple curve $\gamma$ (green, solid) whose mapping through the Rayleigh function $R^{\mathrm{A}}(\gamma)$ is used to determine the number of Rayleigh roots. Branch cuts (red, dashed) and branch points are chosen to warrant depthwise decay of the solution (38)

that is a generalization of Eq.(43) wherein the role of the material parameters is not concealed behind the scaling.

On the real axis,

$$
R_{0}\left(\delta, \lambda_{1}, \lambda_{2}, \eta\right)=\eta^{2} \delta^{4} \geq 0
$$

for the travelling bulk wavenumber $\kappa=\delta$ (or $\kappa=\delta_{1}$ ), while we have the asymptotics

$$
R_{0}\left(\kappa, \lambda_{1}, \lambda_{2}, \eta\right)=-\left(3+2 \eta-\eta^{2}\right) \kappa^{4}+O\left(\kappa^{2}\right), \quad \text { for } \quad \kappa \rightarrow+\infty,
$$

whereupon the Rayleigh function eventually becomes real negative. We conclude, by continuity, that at least a real root is admitted. In fact, we can prove, by the argument principle, that three pairs of central-symmetric roots are present: one real pair corresponding to Rayleigh waves, one purely imaginary pair, corresponding to Rayleigh-like waves, and a third pair of complex conjugated roots, as discussed in [19]. It is observed that in [19] a different choice is made for the cuts, according to which the complex-conjugated pair of zeros may fall outside the Riemann sheet. In fact, with our choice for the cuts, existence of all roots is always warranted.

We determine the number of zeros of (42) in the cut complex plane through the argument principle [2]. To fix ideas, we give the proof for $R^{\mathrm{A}}(\kappa)$, but the argument easily extends to $R^{\mathrm{B}}(\kappa)$. We construct the mapping of the simple curve $\gamma$ through the Rayleigh function, $R^{\mathrm{A}}(\gamma)$, and count its index (or winding number). Looking at Fig.2, we see that $\gamma$ consists of the circle $\gamma_{R}$ of arbitrarily large radius $R$, together with the loops $\gamma_{ \pm \delta}$ around the centrally symmetric pair of cuts $[ \pm \delta, \pm \delta \mp \imath \infty)$ and the loops $\gamma_{ \pm \imath}$ around $[ \pm \imath, \pm \imath \infty)$. By the asymptotics (46), we infer that, when moving along $\gamma_{R}$, the image point makes four complete turns around the origin. We now turn to the loops around the cuts and, in light of the central-symmetry property, only loops sitting in either half-plane are considered and the resulting winding number is then doubled. On the loop $\gamma_{-\imath}$, we have $A_{1}$ and $A_{2}$ purely imaginary, whence Eq.(42) remains in the same form but now in terms of real numbers. In the limit as this loop shrinks down to the cut, $\gamma_{-\imath}$ is mapped onto a open curve approaching the real line from above, i.e. from positive imaginary 
numbers. Conversely, the loop $\gamma_{\delta}$ is mapped onto an S-shaped open curve as in Fig.4, which intersects the real axis three times, named $d_{1}<d_{2}<d_{3}$. In particular, $d_{1}<0$ is located to the left of the origin, while $d_{2}=R^{\mathrm{A}}(\delta)=\delta^{4} \eta^{2} \geq 0$ is always to the right. Together, $D\left(\gamma_{-\imath}\right) \cup D\left(\gamma_{-\imath} \delta\right)$ form a non-simple curve winding once around the origin, that is closed when including the points at infinity. We conclude that six order 1 roots are expected.

Roots should be sought among the zeros of the bi-quartic polynomial

$$
\begin{aligned}
\eta^{4} \kappa^{8}+2(1+\eta) \kappa^{6}+\left(\delta_{2}^{2}-\delta_{1}^{2}\right)(2 \eta+1) \kappa^{4}-\left(2 \eta \delta_{2}^{2} \delta_{1}^{2}+\right. & \left.\delta_{1}^{4}+\delta_{2}^{4}\right) \kappa^{2} \\
& +\delta_{1}^{2} \delta_{2}^{2}\left(\delta_{2}^{2}-\delta_{1}^{2}\right)=0
\end{aligned}
$$

Basically, this is a singularly perturbed polynomial equation inasmuch as $\eta$ is assumed to be small. In this context, we observe that for the case $\eta=0$, corresponding to the strain-gradient theory, Rayleigh waves collapse into bulk waves as Eq.(42) reduces to

$$
\lambda_{1} \lambda_{2}\left(\lambda_{2}^{2}-\lambda_{1}^{2}\right)
$$

whose real roots corresponds to bulk waves $\lambda_{1,2}=0$. In fact, Rayleigh roots are generally perturbations around either bulk wave speed, see [19].

\section{Frequency equation}

For perfect adhesion between the half-spaces at the joining surface $\xi_{2}=0$, we enforce the boundary conditions

$$
\begin{aligned}
w^{\mathrm{A}}(0) & =w^{\mathrm{B}}(0), \\
\frac{\mathrm{d} w^{\mathrm{A}}}{\mathrm{d} \xi_{2}}(0) & =\frac{\mathrm{d} w^{\mathrm{B}}}{\mathrm{d} \xi_{2}}(0), \\
\bar{q}_{1}^{\mathrm{A}}(0) & =\bar{q}_{1}^{\mathrm{B}}(0), \\
p_{3}^{\mathrm{A}}(0) & =p_{3}^{\mathrm{B}}(0) .
\end{aligned}
$$

Plugging the solutions $(38,39)$ into the boundary conditions $(47)$ lends a homogeneous system of linear algebraic equations in the unknown amplitudes $a_{1,2}, b_{1,2}$. This system admits non-trivial solutions inasmuch as the following secular (or frequency) equation is satisfied:

$$
\Delta(\kappa)=0,
$$

in which $\Delta$ is the determinant of the linear system. Introducing the ratio $\Gamma=$ $G^{\mathrm{B}} / G^{\mathrm{A}}$, the determinant in equation (48) may be written as

$$
\Delta(\kappa)=\Gamma \beta^{2}\left(A_{1}-A_{2}\right)\left(B_{1}-B_{2}\right) D_{0}(\kappa),
$$

with

$$
D_{0}(\kappa)=\frac{1}{\Gamma \beta^{2}} R_{0}^{\mathrm{A}}(\kappa)-2 D_{1}(\kappa)+\Gamma \beta^{2} R_{0}^{\mathrm{B}}(\kappa),
$$




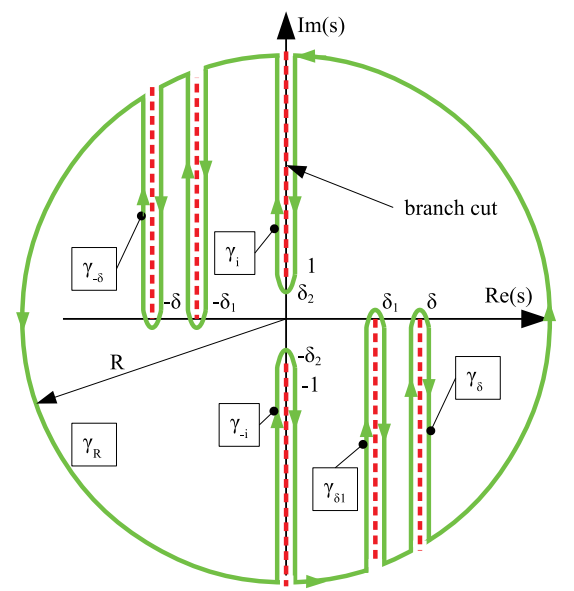

Fig. 3 Simple curve $\gamma$ (green, solid) whose mapping through the Stoneley frequency equation $D_{0}(\gamma)$ is used to determine existence and uniqueness of antiplane Stoneley roots. Here, to fix ideas, we have assumed $\delta_{1}<\delta$ and $\delta_{2}<1$

where $R_{0}^{\mathrm{A}}(\kappa)$ and $R_{0}^{\mathrm{B}}(\kappa)$ are the Rayleigh functions for the relevant half-space and $D_{1}(\kappa)$ is the coupling term. For the latter, we have

$$
\begin{aligned}
D_{1}(\kappa)=\left(\eta^{\mathrm{A}} \kappa^{2}-A_{1} A_{2}\right)\left(\eta^{\mathrm{B}} \kappa^{2}\right. & \left.-B_{1} B_{2}\right) \\
& +\frac{1}{2}\left(A_{1} A_{2}+B_{1} B_{2}\right)\left(A_{1}+A_{2}\right)\left(B_{1}+B_{2}\right) .
\end{aligned}
$$

Here, dependence on $\Omega$ though $\delta, \delta_{1}$ and $\delta_{2}$ is implicitly assumed. Eq.(50) is the CS counterpart of the Rayleigh function [3, Eq.(4-26)], valid for isotropic CE media.

The Rayleigh function (50) exhibits symmetry with respect to $A \leftrightarrow B$ inversion, recalling that we also have $\Gamma \leftrightarrow \Gamma^{-1}$ and $\beta \leftrightarrow \beta^{-1}$. Moreover, when $A=B$, that is

$$
\left(\Gamma, \beta, v, \psi, \eta^{\mathrm{k}}\right)=\left(1,1,1, \delta, \eta^{\mathrm{A}}=\eta^{\mathrm{B}}\right)
$$

we obtain

$$
D_{0, \mathrm{~A}=\mathrm{B}}(\kappa)=-4 A_{1} A_{2}\left(A_{1}+A_{2}\right)^{2},
$$

whence propagation is possible only for $A_{1,2}=0$, that amounts to finding bulk waves. Similarly, in the absence of either half-space, that is for $\Gamma=0$ or $\Gamma \rightarrow \infty$, we find the Rayleigh function of the remaining half-space, that is $R_{0}^{\mathrm{A}}(\kappa)$ or $R_{0}^{\mathrm{B}}(\kappa)$, respectively.

\section{Existence and uniqueness of antiplane Stoneley waves}

We have the asymptotics

$$
D_{0}(\kappa)=-\left(3-\eta^{\mathrm{B}}+\frac{\eta^{\mathrm{A}}+1}{\beta^{2} \Gamma}\right)\left[3-\eta^{\mathrm{A}}+\beta^{2} \Gamma\left(\eta^{\mathrm{B}}+1\right)\right] \kappa^{4}+O\left(\kappa^{2}\right)
$$



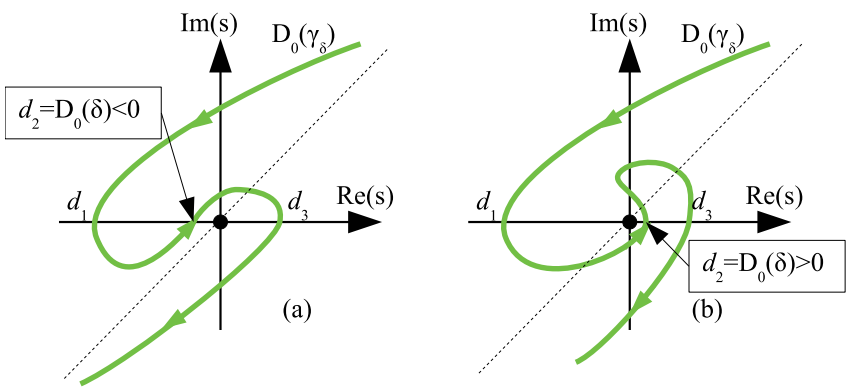

Fig. 4 Image through $D_{0}(s)$ of the loop $\gamma_{\delta}$ for $\Omega<\Omega_{\text {cuton }}$ (left, index $-\frac{1}{2}$ ) and $\Omega>\Omega_{\text {cuton }}$ (right, index $\frac{1}{2}$ ). The dashed line is the common asymptote for the curve at infinity.

whence, on the real axis, the frequency equation eventually becomes negative. For a given triple $\delta, \delta_{1}$ and $\delta_{2}, D_{0}(\kappa)$ is monotonic decreasing and the possibility of a real root for the frequency equation hinges on the fact that

$$
D_{0}\left(\delta_{M}\right) \geq 0, \quad \delta_{M}=\max \left(\delta, \delta_{1}\right) .
$$

This simple analysis may be put into the wider perspective of determining existence and uniqueness of antiplane Stoneley waves. To this aim, we enlarge our viewpoint and think of $D_{0}$ as a function of the complex variable $s$. Then, $D_{0}(s)$ appears centrally symmetric, i.e. $D_{0}(s)=D_{0}(-s)$. We determine the number of zeros of $D_{0}(s)$ in the cut complex plane through the argument principle. Accordingly, we determine the index (winding number) of the curve $D_{0}(\gamma)$, where $\gamma=\gamma_{R} \cup \gamma_{ \pm \delta} \cup$ $\gamma_{ \pm \delta_{1}} \cup \gamma_{ \pm \imath}$ is the simple curve shown in Fig.3. Here, to fix ideas, we assume $\delta_{1}<\delta$ and $\delta_{2}<1$.

When $\Gamma$ is small enough, the following analysis resembles that given for the Rayleigh function. By the asymptotics (52), as the point $\kappa$ moves on the curve $\gamma_{R}$, its mapping $D_{0}(\kappa)$ makes four complete turns about the origin, whence the index is 4 .

As in Fig.4, $\gamma_{\delta}$ is mapped into a open loop having three intersections with the real axis, $d_{1}<0, d_{2}$ and $d_{3}>0$, with $d_{2}=D_{0}(\delta)$. The explicit expression for $d_{2}$ is given in the Appendix. In contrast, $d_{1}$ and $d_{3}$ may be found numerically imposing the condition $\Im\left[D_{0}(\delta \mp \varepsilon-\imath y)\right]=0$, respectively, with $\varepsilon \rightarrow 0^{+}$and $y>0$. When $\Gamma$ is small enough, this loop looks just like the S-shaped curved encountered in the Rayleigh case, but, unlike there, its intersection $d_{2}$ with the real axis is not necessarily positive. Indeed, this loop has index $-\frac{1}{2}$ inasmuch as $d_{2}<0$, that occurs for small values of $\Omega$. In this situation, $D_{0}(s)$ possesses two pairs of roots: a complex-conjugated pair and a purely imaginary pair. Upon increasing $\Omega$, the cuton frequency $\Omega_{\text {cuton }}$ is reached such that $d_{2}=0$ and the real root $\kappa_{S}$ is located precisely at the bulk wavenumber $\delta$. In consideration of the fact that $\delta$ is a monotonic increasing function of $\Omega$ and so is $D_{0}(\Omega)$, for $\Omega>\Omega_{\text {cuton }}$ we have that $D_{0}\left(\gamma_{\delta}\right)$ winds around the origin as in Fig.4(b). Thus, we find three pairs of roots: a complex-conjugated pair, a purely imaginary pair and a real pair.

Similarly, $\gamma_{\delta_{1}}$ is mapped into a loop closed at infinity which never encircles the origin and contribute nothing to the index. Finally, the loop $\gamma_{-\imath}$ is mapped into the real axis from above (i.e. from the side of positive imaginary part) moving 


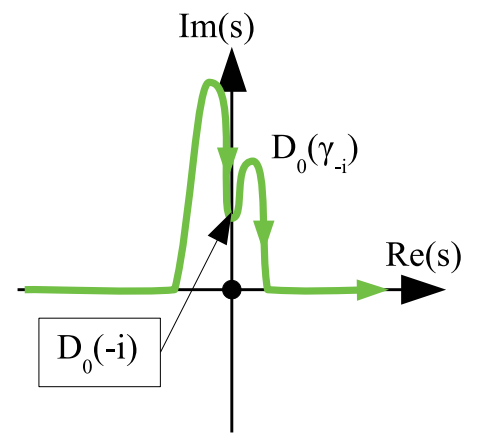

Fig. 5 Image through $D_{0}(s)$ of the loop $\gamma_{-\imath}\left(\right.$ index $\left.\frac{1}{2}\right)$

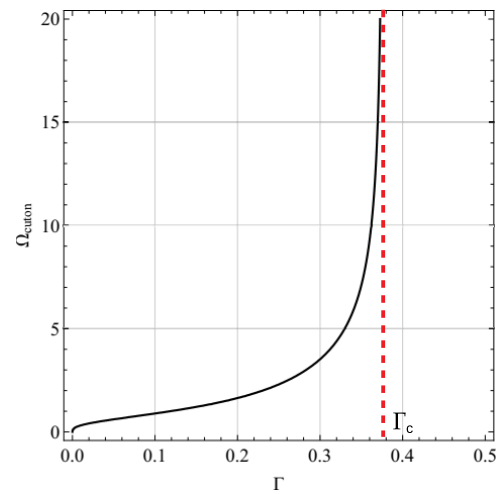

Fig. 6 Cuton frequency as a function of the ratio $\Gamma$ between the shear moduli of media A and $\mathrm{B}$ (solid, black) and vertical asymptote (dashed, red)

from left to right, see Fig.5. This curve brings an index $\frac{1}{2}$ regardless of $\Omega$. We conclude that we have the following scenarios:

- for $\Omega<\Omega_{\text {cuton }}$, the number of roots is 4 , two complex-conjugated, located in the second/fourth quadrant, and two opposite purely imaginary. As discussed in [19], complex roots represent waves decaying/exploding in every direction and have little significance in unbounded media. Conversely, purely imaginary roots represent Stoneley-like waves travelling in the interior of the medium and decaying/exploding along the interface. Such roots are important in semiinfinite situations.

- For $\Omega>\Omega_{\text {cuton }}$, the number of roots is 6 and, alongside the previous four zeros, a pair of real opposite roots, corresponding to travelling Stoneley waves, appears.

Fig.6 shows that the cuton frequency is a monotonic increasing function of $\Gamma$ exhibiting a vertical asymptote. Consequently, a critical value $\Gamma_{c}$ exists for the ratio $\Gamma$, beyond which propagation is blocked. Indeed, for large values of $\Gamma$, the root landscape, as it appears from the argument principle, becomes more involved and, for instance, real (and purely imaginary) roots are eventually lost. A full analysis of all possible scenarios rests outside the scope of this paper. We merely observe that, for a given $\Omega$, the condition (53) demands positivity of a quadratic function of $\Gamma$, 


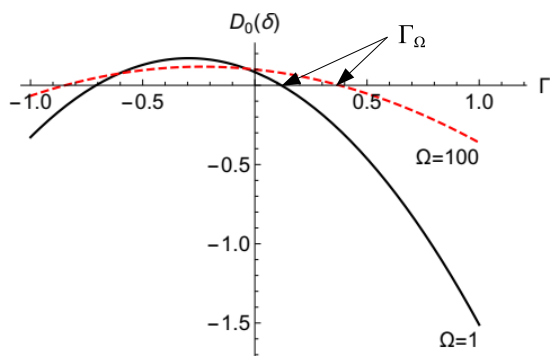

Fig. 7 The root $\Gamma_{\Omega}$ defines the admissible range $\Gamma<\Gamma_{\Omega}$ for propagation of antiplane Stoneley waves to occur at a given frequency: $\Omega=1$ (solid, black) and $\Omega=100$ (dashed, red). We have taken the parameter set: $\ell_{0}^{\mathrm{B}}=0.5, \beta=v=1.1, \eta^{\mathrm{A}}=0.8, \eta^{\mathrm{B}}=0.5$ and $\ell_{0}^{\mathrm{A}}=0.5$

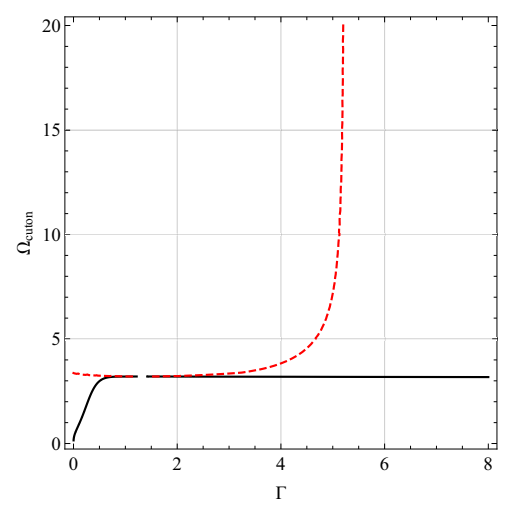

Fig. 8 Cuton frequency as a function of the shear modulus ratio $\Gamma$ for small values of rotational inertia $\left(\ell_{0}^{\mathrm{A}}=0.3\right)$. We see that cuton, as a function of $\Gamma$, possesses a horizontal asymptote and a second branch appears (red, dashed) which is obtained considering $\kappa=\delta_{1}>\delta$ in condition (53)

generally concave upwards, which intersects the $\Gamma$-axis to the right of the origin, in light of (45), at $\Gamma_{\Omega}<\Gamma_{c}$, see Fig.7. As a consequence, a real interval of admissible shear modulus ratios is highlighted, $0<\Gamma<\Gamma_{\Omega}$, which accommodates propagation at and beyond the specified frequency $\Omega$. Fig. 7 supports the observation that this admissible interval increases with $\Omega$ up to the asymptotic value $\Gamma_{c}$. In fact, for large $\Omega$, the quadratic positive real root stabilizes very close to $\Gamma_{c}$.

A different situation develops when rotational inertia in medium A is smaller than medium $\mathrm{B}$, for example, in our parameter set, we consider $\ell_{0}^{\mathrm{A}}=0.3$. Then, $\delta_{1}$ grows with $\Omega$ faster than $\delta$ and eventually overtakes it. This behaviour reflects itself in that the cuton function exhibits a horizontal asymptote at $\Omega_{\text {cuton, asym }} \approx$ 3.20663 , corresponding to this overtaking, see Fig.8. Upon reaching $\Omega_{\text {cuton,asym }}$ from below, the quadratic function of $\Gamma$ eventually reverse convexity and moves above the $\Gamma$-axis: thus, propagation is admitted for any $\Gamma$, as in Fig.9a). Beyond $\Omega_{\text {cuton,asym }}$, the assumption $\delta>\delta_{1}$ is violated: a new admissibility interval may be determined considering the roots $\Gamma_{1} \Omega$ and $\Gamma_{2 \Omega}$ of $D_{0}\left(\delta_{1}\right)$. However, given that convexity has reversed, propagation now occurs outside the interval $\left[\Gamma_{1 \Omega}, \Gamma_{2 \Omega}\right]$. We thus see, in contrast to $\mathrm{CE}$, that propagation of antiplane Stoneley waves is largely possible, even when material properties are significantly different. 

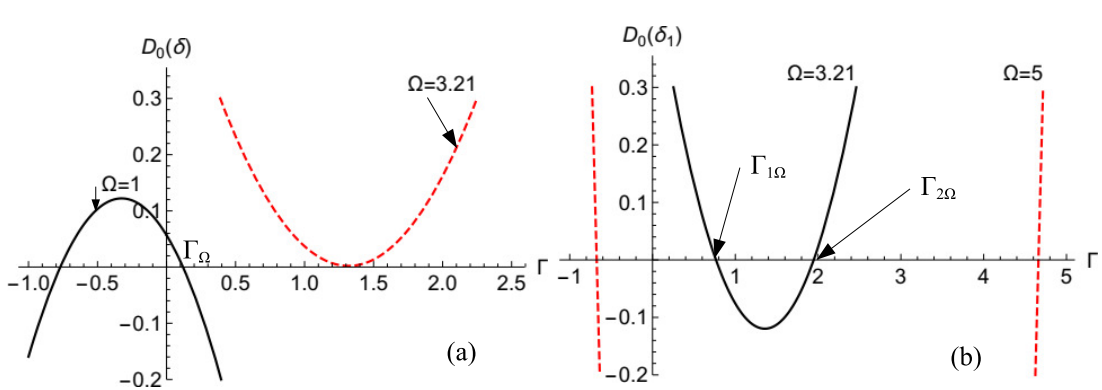

Fig. 9 When rotational inertia of medium $\mathrm{A}, \ell_{0}^{\mathrm{A}}=0.3$, is less than rotational inertia of medium $\mathrm{B}, \ell_{0}^{\mathrm{B}}=0.5$, we find that Stoneley waves propagate (a) within the finite admissible range $\left(0, \Gamma_{\Omega}\right]$ for $\Gamma$ at $\Omega=1$ (solid, black); this interval grows and eventually becomes unbounded upon reaching the asymptotic frequency $\Omega=3.21$ (dashed, red). Beyond this frequency (b), we have $\delta_{1}>\delta$ and admissibility demands $D_{0}\left(\delta_{1}\right) \geq 0$, which sets the unbounded admissibility interval $\left(0, \Gamma_{1 \Omega}\right] \cup\left[\Gamma_{2 \Omega}, \infty\right)$

Finally, we observe that the case $\eta^{\mathrm{A}}=0$ is special, for then intersection with the ordinate axis occurs at the origin, that is a double root for $\Gamma$, i.e. $\Gamma_{\Omega}=0$. However, we have already proved that, in this situation, Stoneley waves collapse into bulk waves.

\section{Dispersion curves}

Travelling wave solutions are possible inasmuch as a set of real solution pairs $\left(\kappa_{S}, \Omega_{S}\right)$ may be found for the frequency equation (49). This is possible in the open interval $\kappa>\delta_{M}$, whence we retrieved the well-known fact, already pointed out in [3], that Stoneley waves are slower than the slowest bulk wave. In our example, $\delta>\delta_{1}$, whence, in light of $(33), \delta_{1}$ is the wavenumber of the fastest bulk wave while, in its neighbourhood, sits the fastest Rayleigh wave $\kappa_{1 R}$. Also, we can show that Stoneley waves are faster than the the slowest Rayleigh wave, whose wavenumber is $\kappa_{R} \gtrsim \delta$. Indeed, looking at (50), we see that, for $\kappa=\kappa_{R}$, the first term drops out (by definition of $\kappa_{R}$ ) and only negative terms remain, in light of the Rayleigh function $R^{\mathrm{B}}(\kappa)$ being monotonic decreasing (and zero at $\kappa_{1 R} \gtrsim \delta_{1}$ ). A solution $\kappa_{S}$ for (50), thought of as a function of $\Gamma$, is possible only inasmuch as $R^{\mathrm{A}}\left(\kappa_{S}\right)>0$, and this occurs only for $\kappa_{S}<\kappa_{R}$. We thus prove the result already observed in [11] and in [15]. Indeed, following the latter, "for all geometries examined [in the context of anisotropic CE], the interface wave velocity is found to lie between the higher free surface (generalized Rayleigh) wave velocity and the slowest bulk wave velocity".

In the following, for the sake of definiteness, when plotting dispersion curves we assume the parameters $\Gamma=0.1, v=\beta=1.1, \ell_{0}^{\mathrm{A}} \leq \ell_{0}^{\mathrm{B}}=0.5$ and $\eta^{\mathrm{B}}=0.5$, whereby bulk (and Rayleigh) waves are faster in B, i.e. $\delta_{1}<\delta$. Fig. 10 presents dispersion curves for Stoneley waves expressing the wavespeed $c_{S}$ as a multiple of the shear bulk wave speed of CE, $c_{\mathrm{s}}^{\mathrm{A}}$, as in Eq.(37).

Similarly to what occurs for Rayleigh waves [20, 19], Stoneley waves are initially dispersive, yet they soon develop a horizontal asymptote that is located above (below) the shear wave speed of CE, according to $\ell_{0}^{\mathrm{A}} \gtrless \ell_{0 \mathrm{cr}}^{\mathrm{A}}$. Therefore, the dispersive nature of the wave is restricted to small wavenumbers. However, 


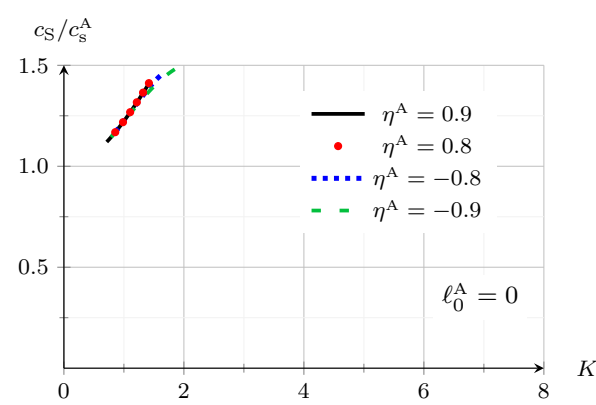

(a)

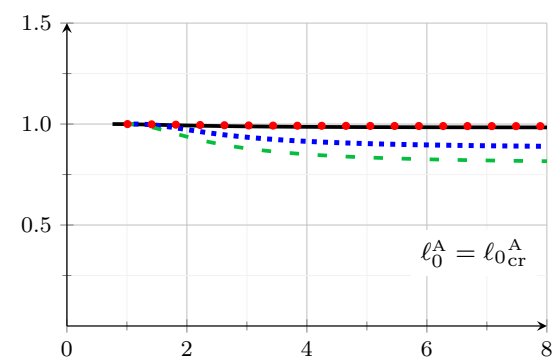

(c)

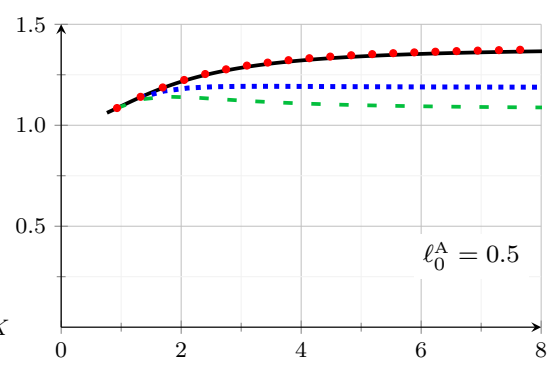

(b)

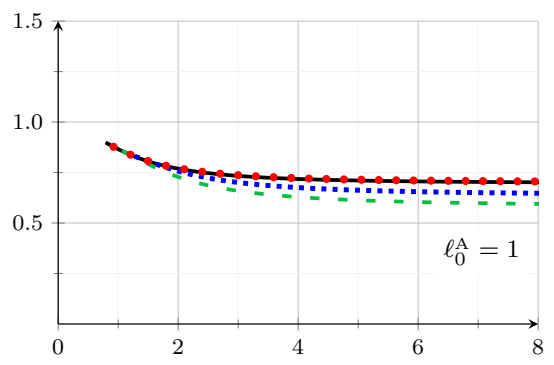

(d)

Fig. 10 Dispersion curves for Stoneley waves where the phase speed $c_{\mathrm{S}}$ is expressed as a multiple of $c_{\mathrm{s}}^{\mathrm{A}}$, that is the $\mathrm{SH}$ bulk wave speed of classical elasticity for medium $\mathrm{A}(\Gamma=0.1$, $v=\beta=1.1, \ell_{0}^{\mathrm{B}}=0.5$, and $\left.\eta^{\mathrm{B}}=0.5\right)$

in the absence of rotational inertia in the half-space A (recall $\Gamma$ is small), that is for $\ell_{0}^{\mathrm{A}}=0$ (see Fig.10a)), the curve is monotonic increasing and dispersion is always warranted. As already discussed, bulk nondispersive waves, moving with the constant speed $c_{s}^{\mathrm{A}}$, are found for either $\eta^{\mathrm{A}}=0$ or $\ell_{0}^{\mathrm{A}}=\ell_{0 \mathrm{cr}}^{\mathrm{A}}$.

So far, the behaviour of Stoneley waves is very similar to that of Rayleigh waves, see $[20, \S 5]$, of which they are perturbations through $\Gamma$. However, in contrast to Rayleigh waves, a cuton frequency is met below which propagation is prevented. Therefore, Stoneley waves present a zero-frequency block-band and their propagation follows an optical branch. Fig.11, illustrates the role of $\Gamma$ and $\eta^{\mathrm{A}}$ in determining the cuton frequency. Also, propagation is possible below a critical value $\Gamma_{\Omega}\left(\right.$ case $\left.\delta>\delta_{1}\right)$ and outside the finite interval $\Gamma_{1 \Omega}<\Gamma<\Gamma_{2 \Omega}\left(\right.$ case $\left.\delta_{1}>\delta\right)$, as discussed in $\S 6$. Especially, in stark contrast to CE, Stoneley wave propagation occurs in CS elasticity under pretty general conditions, well beyond Wiechert's conditions, and they appear to be the rule rather than the exception.

Still, similarly to Rayleigh waves, Stoneley waves are perturbations of the travelling bulk modes. Indeed, following [19], a convenient approach to the determination of Stoneley wavenumbers is obtained expanding Eq.(48) around $\delta$ (or $\delta_{1}$, depending which is the largest) through setting

$$
\kappa_{s}=\delta\left(1+\epsilon_{S}^{2}\right), \quad \epsilon_{S} \ll 1
$$




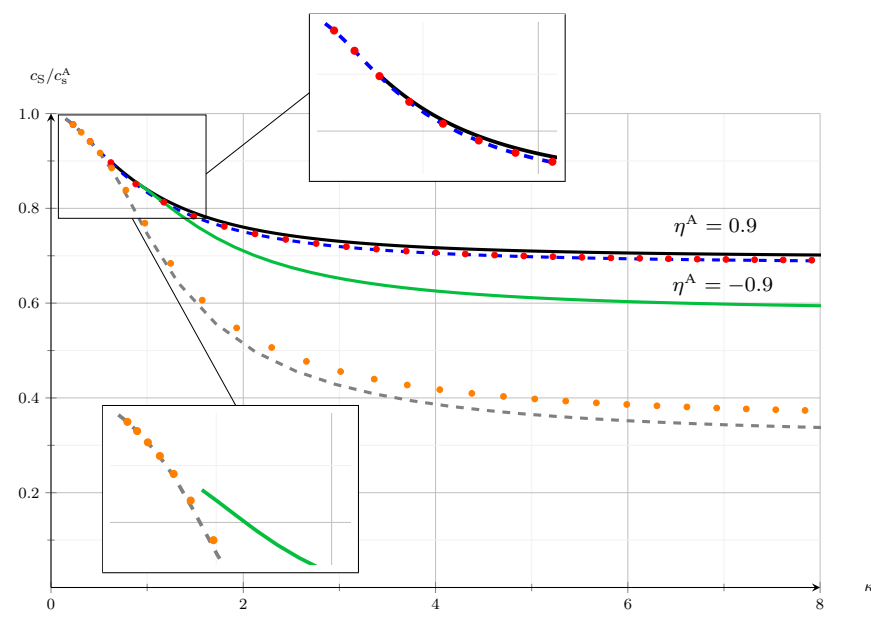

Fig. 11 Dispersion curves for antiplane Stoneley waves at $\Gamma=0.1$ (solid), $\Gamma=0.01$ (dots), and $\Gamma=0.001$ (dashed)

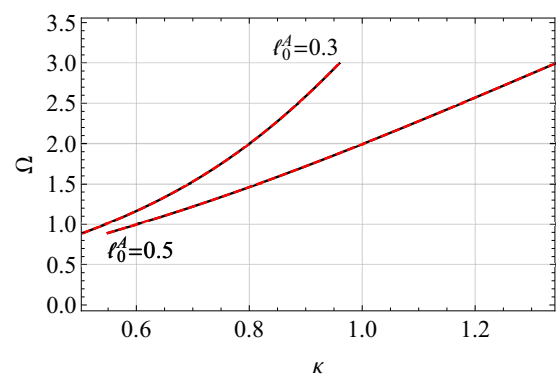

Fig. 12 Linear approximation (54) (dashed, red) superposed onto the numerically evaluated frequency spectrum (solid, black) for $\ell_{0}^{\mathrm{A}}=0.3$ and $\ell_{0}^{\mathrm{A}}=0.5$. Curves perfectly overlap in the considered domain.

Solutions of the resulting linear equation in $\epsilon_{S}$

$$
d_{2}+a_{1} \epsilon_{S}=0
$$

are plotted in Fig.12 against the numerically evaluated frequency spectra. It is seen that approximated and "exact" spectra perfectly overlap in the whole range considered. The coefficients $d_{2}$ and $a_{1}$ are given in the Appendix.

\section{Conclusions}

Propagation of antiplane Stoneley waves is investigated within the context of couple stress theory, in an attempt to discuss the role of material microstructure in developing new pathways for energy transport. Remarkably, it is found that antiplane Stoneley waves are supported under rather general conditions, and this outcome stands in marked contrast with the findings of classical elasticity, wherein antiplane Stoneley waves are not supported altogether and in-plane Stoneley waves 
are possible only for a very restricted range for the ratio between the material constants of the media in contact. In fact, according to Wiechert's conditions, material pairs supporting in-plane Stoneley waves within classical elasticity ought to be very similar.

The question of existence and uniqueness of such waves is also addressed by the argument principle. It is found that, besides travelling waves, evanescent and decaying/exploding modes are also admitted, in a complex wave pattern. Interestingly, propagation is possible only beyond a cuton frequency, for which an explicit expression is given. Indeed, it appears that lack of propagation, typical of classical elasticity, is relaxed to high-frequency propagation by the presence of the microstructure. As a result, rotational inertia plays an important role as it affects the admissibility range for propagation.

Stoneley waves, just like Rayleigh waves, are perturbations of the relevant bulk modes. As a result, an approximated linear (in the wavenumber) expression for locating Stoneley roots is given that proves extremely accurate when compared to plain numerics in a wide frequency range. The possibility of Stoneley wave propagation under general conditions has great importance in seismology and it is a valuable asset in non-destructive testing of materials.

\section{Declarations}

9.1 Funding

This research was supported under the grant POR FESR 2014-2020 ASSE 1 AZIONE 1.2.2 awarded to the project "IMPReSA" CUP E81F18000310009

\subsection{Conflicts of interest/Competing interests}

Authors declare no competing interests.

9.3 Availability of data and material

This paper contains no data.

9.4 Code availability

Not applicable.

Acknowledgements Enrico Radi's help in simplifying the dispersion relation is gratefully acknowledged. 


\section{A Linear approximation to cuton}

In this short Appendix we gather the analytic expressions of the coefficients in the linear approximation (54). They are

$$
\begin{gathered}
d_{2}=\beta^{2} \Gamma\left[\left(\sqrt{\left(\delta^{2}-\delta_{1}^{2}\right)\left(\delta^{2}+\delta_{2}^{2}\right)}-\delta^{2} \eta^{\mathrm{B}}\right)^{2}\right. \\
\left.\quad-\sqrt{\left(\delta^{2}-\delta_{1}^{2}\right)\left(\delta^{2}+\delta_{2}^{2}\right)}\left(\sqrt{\delta^{2}-\delta_{1}^{2}}+\sqrt{\delta^{2}+\delta_{2}^{2}}\right)^{2}\right] \\
+\frac{\delta^{4}\left(\eta^{\mathrm{A}}\right)^{2}}{\beta^{2} \Gamma}-2 \delta^{4} \eta^{\mathrm{A}} \eta^{\mathrm{B}}+2 \sqrt{\left(\delta^{2}-\delta_{1}^{2}\right)\left(\delta^{2}+\delta_{2}^{2}\right)} \delta^{2} \eta^{\mathrm{A}} \\
-\sqrt{\left(\delta^{2}+1\right)\left(\delta^{2}-\delta_{1}^{2}\right)\left(\delta^{2}+\delta_{2}^{2}\right)}\left(\sqrt{\delta^{2}-\delta_{1}^{2}}+\sqrt{\delta^{2}+\delta_{2}^{2}}\right),
\end{gathered}
$$

and

$$
\begin{aligned}
& a_{1}=\frac{\sqrt{2} \delta}{\beta^{2} \Gamma}\left\{\delta ^ { 2 } \left[-2 \beta^{2} \Gamma\right.\right.\left.\left(\sqrt{\delta^{2}-\delta_{1}^{2}}+\sqrt{\delta^{2}+\delta_{2}^{2}}-\eta^{\mathrm{B}} \sqrt{\delta^{2}+1}\right)-\sqrt{\delta^{2}+1}\left(2 \eta^{\mathrm{A}}+1\right)\right] \\
&-\beta^{2} \Gamma\left[\sqrt{\delta^{2}-\delta_{1}^{2}} \delta_{2}^{2}+\sqrt{\delta^{2}-\delta_{1}^{2}}-\delta_{1}^{2} \sqrt{\delta^{2}+\delta_{2}^{2}}\right. \\
&\left.\left.+\sqrt{\delta^{2}+\delta_{2}^{2}}+2 \sqrt{\left(\delta^{2}+1\right)\left(\delta^{2}-\delta_{1}^{2}\right)\left(\delta^{2}+\delta_{2}^{2}\right)}\right]-\sqrt{\delta^{2}+1}\right\} .
\end{aligned}
$$

Naturally, in the special case $\Gamma \rightarrow 0$, we retrieve the result already obtained for Rayleigh waves in [19].

\section{References}

1. VTN Anh, LT Thang, PC Vinh, and TT Tuan. Stoneley waves with spring contact and evaluation of the quality of imperfect bonds. Zeitschrift für angewandte Mathematik und Physik, 71(1):36, 2020.

2. AF Beardon. Complex analysis: The argument principle in analysis and topology. Courier Dover Publications, 2019.

3. L Cagniard. Reflection and refraction of progressive seismic waves. McGraw-Hill, 1962.

4. P Chadwick and P Borejko. Existence and uniqueness of stoneley waves. Geophysical Journal International, 118(2):279-284, 1994.

5. G Dal Moro. Surface wave analysis for near surface applications. Elsevier, 2014.

6. V A Eremeyev, G Rosi, and S Naili. Surface/interfacial anti-plane waves in solids with surface energy. Mechanics Research Communications, 74:8-13, 2016.

7. $\mathrm{H}$ Fan and $\mathrm{L} \mathrm{Xu}$. Love wave in a classical linear elastic half-space covered by a surface layer described by the couple stress theory. Acta Mechanica, 229(12):5121-5132, 2018.

8. HG Georgiadis and EG Velgaki. High-frequency Rayleigh waves in materials with micro-structure and couple-stress effects. International Journal of Solids and Structures, 40(10):2501-2520, 2003.

9. PA Gourgiotis and HG Georgiadis. Torsional and SH surface waves in an isotropic and homogenous elastic half-space characterized by the Toupin-Mindlin gradient theory. International Journal of Solids and Structures, 62:217-228, 2015.

10. PA Gourgiotis, HG Georgiadis, and I Neocleous. On the reflection of waves in halfspaces of microstructured materials governed by dipolar gradient elasticity. Wave Motion, 50(3):437-455, 2013.

11. TM Hsieh, EA Lindgren, and M Rosen. Effect of interfacial properties on Stoneley wave propagation. Ultrasonics, 29(1):38-44, 1991.

12. AV Ilyashenko. Stoneley waves in a vicinity of the wiechert condition. International Journal of Dynamics and Control, pages 1-3, 2020. 
13. WT Koiter. Couple-stress in the theory of elasticity. In Proc. K. Ned. Akad. Wet, volume 67, pages 17-44. North Holland Pub, 1964.

14. R Kumar, S Devi, and SM Abo-Dahab. Stoneley waves at the boundary surface of modified couple stress generalized thermoelastic with mass diffusion. Journal of Applied Science and Engineering, 21(1):1-8, 2018.

15. TC Lim and MJP Musgrave. Stoneley waves in anisotropic media. Nature, 225(5230):372$372,1970$.

16. AE Love. Some problems of geodynamics, 1911.

17. R D Mindlin. Micro-structure in linear elasticity. Archive for Rational Mechanics and Analysis, 16(1):51-78, 1964.

18. G Mishuris, A Piccolroaz, and E Radi. Steady-state propagation of a mode III crack in couple stress elastic materials. International Journal of Engineering Science, 61:112-128, 2012.

19. A Nobili, E Radi, and C Signorini. A new Rayleigh-like wave in guided propagation of antiplane waves in couple stress materials. Proceedings of the Royal Society A, 476(2235):20190822, 2020.

20. A Nobili, E Radi, and A Vellender. Diffraction of antiplane shear waves and stress concentration in a cracked couple stress elastic material with micro inertia. Journal of the Mechanics and Physics of Solids, 124:663-680, 2019.

21. B Noble. Methods based on the Wiener-Hopf technique for the solution of partial differential equations, International Series of Monographs on Pure and Applied Mathematics. Vol. 7. Pergamon Press, New York, 1958.

22. N S Ottosen, M Ristinmaa, and C Ljung. Rayleigh waves obtained by the indeterminate couple-stress theory. European Journal of Mechanics-A/Solids, 19(6):929-947, 2000.

23. TE Owen. Surface wave phenomena in ultrasonics. Progress in Applied Material Research, 6:71-87, 1964

24. E Radi. On the effects of characteristic lengths in bending and torsion on mode III crack in couple stress elasticity. International Journal of Solids and Structures, 45(10):3033-3058, 2008.

25. Sl Rokhlin, M Hefets, and M Rosen. An elastic interface wave guided by a thin film between two solids. Journal of Applied Physics, 51(7):3579-3582, 1980.

26. JG Scholte. The range of existence of rayleigh and stoneley waves. Geophysical Supplements to the Monthly Notices of the Royal Astronomical Society, 5(5):120-126, 1947.

27. HM Shodja, A Goodarzi, MR Delfani, and H Haftbaradaran. Scattering of an anti-plane shear wave by an embedded cylindrical micro-/nano-fiber within couple stress theory with micro inertia. International Journal of Solids and Structures, 58:73-90, 2015.

28. R Stoneley. Elastic waves at the surface of separation of two solids. Proceedings of the Royal Society of London. Series A, Containing Papers of a Mathematical and Physical Character, 106(738):416-428, 1924.

29. R Stoneley. Rayleigh waves in a medium with two surface layers.(first paper). Geophysical Supplements to the Monthly Notices of the Royal Astronomical Society, 6(9):610-615, 1954.

30. JW Strutt. On waves propagated along the plane surface of an elastic solid. Proceedings of the London Mathematical Society, 17(1):4-11, 1885.

31. PC Vinh and PTH Giang. On formulas for the velocity of stoneley waves propagating along the loosely bonded interface of two elastic half-spaces. Wave Motion, 48(7):647-657, 2011.

32. PC Vinh, PG Malischewsky, and PTH Giang. Formulas for the speed and slowness of Stoneley waves in bonded isotropic elastic half-spaces with the same bulk wave velocities. International Journal of Engineering Science, 60:53-58, 2012.

33. FACM Yang, ACM Chong, DCC Lam, and P Tong. Couple stress based strain gradient theory for elasticity. International Journal of Solids and Structures, 39(10):2731-2743, 2002.

34. L Zhang, Y Huang, JY Chen, and KC Hwang. The mode III full-field solution in elastic materials with strain gradient effects. International Journal of Fracture, 92(4):325-348, 1998.

35. T Zisis. Anti-plane loading of microstructured materials in the context of couple stress theory of elasticity: half-planes and layers. Archive of Applied Mechanics, 88(1-2):97-110, 2018. 